OPEN ACCESS

Edited by:

Jiarui Wu,

Beijing University of Chinese

Medicine, China

Reviewed by:

Jianye Dai,

Lanzhou University, China

Haiyang $Y u$,

Tianjin University of Traditional

Chinese Medicine, China

Xinkui Liu,

Beijing University of Chinese

Medicine, China

*Correspondence:

Daiyin Peng

pengdaiyin@163.com

Specialty section:

This article was submitted to

Ethnopharmacology,

a section of the journal

Frontiers in Pharmacology

Received: 06 November 2019

Accepted: 13 December 2019

Published: 24 January 2020

Citation:

Duan X, Pan L, Bao Q and Peng D (2020) UPLC-Q-TOF-MS Study of the

Mechanism of THSWD for Breast

Cancer Treatment.

Front. Pharmacol. 10:1625.

doi: 10.3389/fphar.2019.01625

\section{UPLC-Q-TOF-MS Study of the Mechanism of THSWD for Breast Cancer Treatment}

\author{
Xianchun Duan ${ }^{1,2}$, Lingyu Pan ${ }^{1}$, Qiuyu Bao ${ }^{2}$ and Daiyin Peng ${ }^{2 *}$ \\ ${ }^{1}$ Department of Pharmacy, The First Affiliated Hospital of Anhui University of Traditional Chinese Medicine, Hefei, China, \\ ${ }^{2}$ Anhui Province Key Laboratory of Chinese Medicinal Formula, Synergetic Innovation Center of Anhui Authentic Chinese \\ Medicine Quality Improvement, Anhui University of Chinese Medicine, Hefei, China
}

Taohong Siwu decoction (THSWD) is a classic traditional Chinese medicine (TCM) prescription that is widely used in the clinical treatment of gynecological and cerebrovascular diseases. Here we used a method that coupled ultra-performance liquid chromatography to quadrupole time-of-flight mass spectrometry (UPLC-Q-TOF$\mathrm{MS}$ ) in which both positive and negative ion modes were established to investigate the major constituents in THSWD. A Waters ACQUITY UPLC BEH C18 column $(2.1 \mathrm{~mm} \times 100$ $\mathrm{mm}, 1.7 \mu \mathrm{m}$ ) was used to separate the aqueous extract of THSWD. The mobile phase consisted of $0.1 \%$ aqueous formic acid (A) and acetonitrile (B). Ninety-five components were identified in two different ion modes, including aromatic acids, flavones, polysaccharides, volatile oils monoterpene glycosides, aromatic cyanogenic glycosides, and others. Pathological changes in tumors and serum expression of interleukin-4 in a mouse model of breast cancer were detected after THSWD treatment. The results showed that THSWD had obvious therapeutic effects. This study establishes a material basis for the use of THSWD in the treatment of breast cancer.

Keywords: Taohong Siwu decoction, breast cancer, UPLC-Q-TOF-MS, components, rats

\section{INTRODUCTION}

Traditional Chinese medicine (TCM) is an important part of Chinese culture (Zhen, 2013). As the most representative and integral part of traditional Chinese culture, TCM has a long, profound history. At present, the Party and State attach great importance to TCM, which also has a far-reaching influence all over the world (Weiwei, 2017). Formulae are the main form of TCM clinical medicine (Chen and Luo, 2018). They are chara+cterized by multiple components, which affect multiple pathways and targets, so their mechanisms of action are largely unknown (Li et al., 2012). The "Traditional Chinese Medicine Law of the People's Republic of China" is its official implementation, marking the first time that China identified the important status, development guidelines, and supporting measures of TCM from a legal perspective and provided a legal guarantee for TCM development.

Taohong Siwu decoction (THSWD) consists of six species of medicinal herbs: Prunus persica (L.) Batsch (Taoren, TR), Carthamustinctorius L. (Honghua, HH), Angelica sinensis (Oliv.) Diels (Danggui, DG), Ligusticum chuanxiong hort (Chuanxiong, CX), Paeoniae Radix Alba (Baishao, BS), and Rehmanniaglutinosa (Gaertn.) DC. (Shudi, SD) (Ding and Zhang, 2010). THSWD was 
documented in "Yi Zong Jin Jian" compiled by Wu Qian in the Qing Dynasty, on the basis that the Siwu decoction enriches the blood, adds TR and HH into the blood, eliminates blood stasis, and promotes blood circulation and nourishment (Liu et al., 2015). It has been widely used in the treatment of gynecological and cerebrovascular diseases (Kim et al., 2016). Ischemic stroke, also known as cerebral infarction due to thrombosis, belongs to the category of "stroke" in TCM (Yu et al., 2007). It is an acute cerebrovascular disease characterized by sudden stupor, unconsciousness, hemiplegia, slurred speech, and speech disorder (Cheng et al., 2018).

Considering the characteristics of a TCM compound, the composition is complex. Moreover, factors like origin, cultivation, harvesting, processing, preparation, and more all influence the clinical treatment effect (Zhang, 2010). Therefore, verifying the chemical composition of TCM compounds through analysis and quality testing is particularly important. It is quite time-consuming to chemically profile TCM preparations with conventional high-performance liquid chromatography coupled with ultraviolet detection (HPLC-UV) or HPLC coupled with mass spectrometry (HPLC-MS) (Han et al., 2007; Li et al., 2014). Compared with traditional HPLC technology, which remains constant over a wider linear range, ultra-performance liquid chromatography (UPLC) has higher efficiency. Its advantages include increasing the mobile phase velocity, shortening the analysis time, and increasing throughput. The columns show superior performance, and the ultra-high pressure liquid chromatography pump offers precise gradient control, low diffusion, a low cross-contamination auto-sampler system, a high-speed detector, and comprehensive hardware and software. The peak capacity, analysis efficiency, and sensitivity are greatly improved compared with conventional HPLC, providing a good platform for the separation analysis of complex systems (Gumustas et al., 2014; Hedaya et al., 2017; Wu et al., 2017). Time-of-flight MS (TOF-MS) can provide accurate molecular mass (Zhang et al., 2018). MassHunter software can calculate the molecular formula of the test substance, so the chemical composition represented by each chromatographic peak can be directly identified with very small errors, generally at $5 \mathrm{ppm}$. This approach is widely used in the identification of compounds. In this study, UPLC-Q-TOF-MS technology was used to separate and identify the main components of THSWD.

THSWD is used to treat cerebrovascular and gynecological diseases. DG and CX have good therapeutic effects on gynecological conditions, especially breast diseases. However, there have been no reports of the study of THSWD in the treatment of breast cancer. We analyzed the components of THSWD and assessed its therapeutic effect in a mouse model of breast cancer to provide a basis for its clinical use.

\section{MATERIALS AND METHODS}

\section{Materials}

Prunus persica (L.) Batsch (Taoren, TR, batch number: 17033101), Carthamus tinctorius L. (Honghua, HH, batch number: 17041401), Angelica sinensis (Oliv.) Diels (Danggui, DG, batch number: 16070501), Conioselinum anthriscoides 'Chuanxiong' (syn. Ligusticum chuanxiong Hort) (Chuanxiong, CX, batch number: 17061601), Paeoniae lactiflora Pall. (Baishao, BS, batch number: 17050301), and Rehmannia glutinosa (Gaertn.) DC (Shudi, SD, batch number: 17042501) were purchased from Anqing Huashi Chinese Herbal Medicine Co. Ltd. (Anqing, China). All TCM materials were qualified by Professor Huasheng Peng (hspeng@126.com). All voucher specimens were deposited at the Herbarium of Anhui University of Chinese Medicine, Hefei, China (Herbarium code: ACM, voucher numbers: 17021, 17025, 17034, 17051, 17068, 17080). A Waters ACQUITYTM UPLC I-Class and a SYNAPTG2-Si MS were obtained from Waters Corporation (Milford, MA, USA). The 4T1 breast cancer cell line and fetal bovine serum (batch number: 20160720) were from Zhejiang Tianhang Biotechnology Co., Ltd. RPMI 1640 medium (batch number: ZI110516) was purchased from Hangzhou Northrend Biotechnology Co., Ltd.

\section{Preparation and Extraction}

In this system, the herbs TR, HH, DG, SD, CX, and BS (3:2:3:4:2:3) were soaked then decocted twice with 10 vol. of boiling water for $2 \mathrm{~h}$ and 8 vol. of boiling water for $1.5 \mathrm{~h}$. The extraction solutions were then filtered and combined. Next, 95\% ethanol was added for alcohol precipitation to obtain TaohongSiwu decoction alcohol precipitation. A small amount of alcohol solution was filtered through a $0.22-\mu \mathrm{m}$ microporous membrane filter, and $2 \mu \mathrm{L}$ of filtrate was used for injection analysis.

\section{UPLC-MS and UPLC-MS ${ }^{2}$ Analysis}

A Waters ACQUITY UPLC BEH C18 column (2.1 mm×100 $\mathrm{mm}, 1.7 \mu \mathrm{m})$ was used to separate the aqueous extract of THSWD. The mobile phase was $0.1 \%$ aqueous formic acid (A) and acetonitrile (B). Chromatographic separation was performed at $35^{\circ} \mathrm{C}$ in this system. Gradient elution with a flow rate of $0.3 \mathrm{~mL} / \mathrm{min}$ was performed as follows: $3 \% \mathrm{~B}$ at $0-2 \mathrm{~min}$; $3 \%-8 \% \mathrm{~B}$ at $2-8 \mathrm{~min} ; 8 \%-25 \% \mathrm{~B}$ at $8-12 \mathrm{~min} ; 25 \%-25 \% \mathrm{~B}$ at $12-15$ $\min ; 25 \%-45 \%$ B at $15-16 \mathrm{~min} ; 45 \%-90 \%$ B at $16-22$ min; $90 \%-$ $100 \% \mathrm{~B}$ at $22-26 \mathrm{~min} ; 100 \% \mathrm{~B}$ at $26-28 \mathrm{~min}$. The MS analysis was carried out with the electrospray ionization (ESI) source in both positive and negative ion modes, and leucine enkephalin was used as the accurate mass calibration solution. The desolvation gas temperature was $350^{\circ} \mathrm{C}$. The flow rates of cone and desolvation gases were set at $50 \mathrm{~L} / \mathrm{h}$ and $600 \mathrm{~L} / \mathrm{h}$, respectively. The capillary, cone, and extraction cone voltages were set to 3.0 and $2.5 \mathrm{~V}$ in positive and negative ion modes, respectively. $\mathrm{MS}^{\mathrm{E}}$ was applied for MS/MS analysis with a low collision energy of 6 $\mathrm{V}$ and a high collision energy of $20-80 \mathrm{~V}$. The scan area was set at $m / z 50-1200$.

\section{Animal Treatment and Sample Collection}

$\mathrm{BALB} / \mathrm{c}$ mice (specific pathogen-free grade, $22 \pm 2 \mathrm{~g}$ ), were purchased from the Laboratory Animal Center, Medical University of Anhui Province (permit number: 2016AH-031-11). They were randomly divided into six groups (10 mice each): normal; model; THSWD low-dose, middle-dose, and high-dose 
(L, M, and H); and cisplatin. After 4T1 cells were resuscitated, they were routinely cultured in RPMI 1640 medium containing 10\% fetal bovine serum at $5 \% \mathrm{CO}_{2}$ and $37^{\circ} \mathrm{C}$. The culture medium was changed every 2 to 3 days and passaged. Cells cultured to logarithmic growth phase were collected and diluted 10 times with sterile saline, and $0.2 \mathrm{~mL}$ was injected into each mouse. After 48 hours, there was a small pink protrusion of about $2 \mathrm{~mm} \times$ $2 \mathrm{~mm}$, indicating successful modeling. Mice in the THSWD $\mathrm{H}$, THSWD M, and THSWD L groups were treated with THSWD $(25.2,12.6$, and $6.3 \mathrm{~g} / \mathrm{kg}$, respectively, three times a day) by gavage. The cisplatin group received cisplatin $(0.1 \mathrm{mg} / \mathrm{mL})$ by gavage, the normal and model groups were administered with an equivalent amount of regular saline. The dosing period was 18 days. After the last administration, blood and tumor samples were collected and animals were sacrificed by cervical vertebrae dislocation. All experiments were subject to approval by the Committee on the Ethics of Animal Experiments of Anhui University of Chinese medicine (Permit Number: LLSC20160336).

\section{Hematoxylin and Eosin (H\&E) Staining}

Tumor tissues were fixed, paraffin-embedded, and sectioned. Changes in tumor cell arrangement and pathological alterations of the tumor were observed with conventional H\&E staining and analyzed using ImageJ software (National Institutes of Health, Bethesda, MD, USA).

\section{Interleukin (IL)-4, IL-10, IL-13, and Transforming Growth Factor (TGF)- B1 Expression}

Serum was collected from mice and centrifuged at $350 \mathrm{~g}$ for 5 min at $4^{\circ} \mathrm{C}$. Supernatants were stored at $-80^{\circ} \mathrm{C}$ for cytokine analysis. Enzyme-linked immunosorbent assays (ELISAs) were performed to quantify the concentrations of IL-4, IL-10, IL-13, and TGF- $\beta 1$, according to the manufacturer's instructions. Optical density (OD) values were read at $450 \mathrm{~nm}$, and the levels of IL-4, IL-10, IL-13, and TGF- $\beta 1$ were determined based on standard curves.

\section{CD44, CD117, and CD133 Expression}

At 24 hours after transfection, the cells were removed and washed with phosphate-buffered saline (PBS; $5 \mathrm{~min}, 3$ times), fixed in methanol $\left(-20^{\circ} \mathrm{C}, 5 \mathrm{~min}\right)$, fixed in $70 \%$ ethanol for $5 \mathrm{~min}$, and washed with PBS (5 min, 3 times). They were then blocked in $1 \%$ skimmed milk powder for $30 \mathrm{~min}$, and the mixture was incubated for $2 \mathrm{~h}$ at room temperature with primary antibody. The cells were washed with PBS, incubated with secondary antibody for $1 \mathrm{~h}$, stained with 4',6-diamidino-2-phenylindole for 2 min, washed with PBS (5 min, 3 times), mounted in DAKO medium (DAKO, Glostrup, Denmark), observed under a fluorescent microscope, and photographed.

\section{Data Processing}

\section{Establishment of a Component Analysis Database}

We used the UNIFI software library management system to collect data from offline and online MS databases such as PubMed (http://www.ncbi.nlm.nih.gov/pubmed), MassBank (http://www.massbank.jp/), Chemspider (http://www. chemspider.com/), and METLIN (https://en.wikipedia.org/) and identify relevant literature on the chemical composition of THSWD. We collected the names and molecular and structural formulas to build a database of the chemical constituents of THSWD.

\section{Data Acquisition and Analysis}

The $\mathrm{MS}^{\mathrm{E}}$ Continuum model was used to collect data and determine the chemical composition of THSWD, which was automatically identified by the UNIFI data processing system. MassLynx 4.1 software (Waters Corporation) was used to deal with the base-peak ion current patterns of positive and negative ion modes. The main chemical components were identified and confirmed by the accurate mass of fragment ions theory, relative retention time, offline and online mass spectral database, and related literature.

\section{RESULTS AND DISCUSSION}

\section{Optimization of Chromatographic Conditions and Q-TOF-MS/MS Method Development}

Due to the diversity of TCM prescriptions, the complexity of each single herb, and the large variety of structures, it is necessary to optimize the chromatographic conditions to obtain and separate the compounds quickly and improve the resolution. The items to be improved are the mobile phase, flow rate, column temperature, and MS parameters. For the mobile phase, we chose aqueous formic acid (A) and acetonitrile (B). We set the column temperature at $35^{\circ} \mathrm{C}$. The detection wavelength was based on the literature, and full-band scans were performed at $320 \mathrm{~nm}$ with a flow rate of $0.3 \mathrm{~mL} / \mathrm{min}$ to reduce the column pressure and shorten the analytical time. The elution procedures and MS conditions were set according to section 2.3. The standard sample solution and medicinal sample solution of each ingredient in THSWD were precisely absorbed. The total ion chromatogram of the THSWD solution was obtained according to the liquid-phase and MS conditions and the injection analysis. The base peak chromatograms of THSWD in positive and negative ion modes are shown in Figures $\mathbf{1}$ and 2 , respectively.

\section{Analysis of THSWD by UPLC-Q-TOF-MS and Identification of Its Main Constituents}

The molecular weights of the 87 peaks extracted from the total ion chromatogram of the extract of THSWD were consistent with the data of the previously built pool, including aromatic acids, flavones, polysaccharides, volatile oils, monoterpene glycosides, aromatic cyanogenic glycosides, and others (Table 1 and Table 2). Their sources were confirmed by comparing the base peak chromatograms of THSWD with its six separate herbal extracts. Standard compounds available were identified by comparing retention time with the exact mass. For 


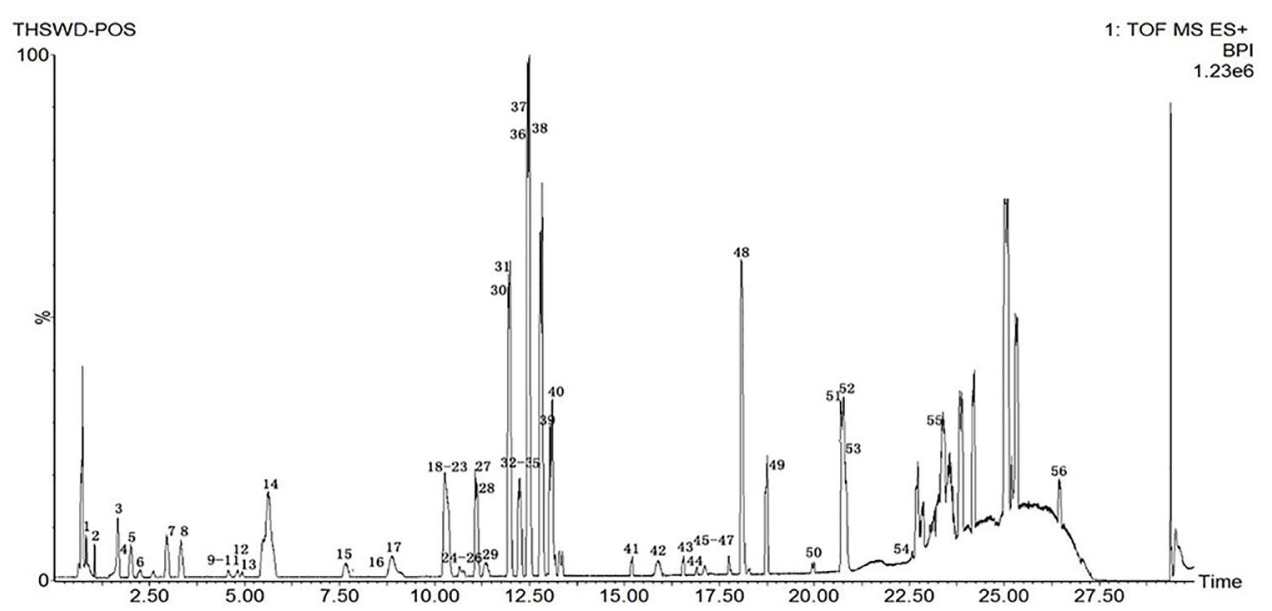

FIGURE 1 | Base peak chromatograms of THSWD in positive ion mode.

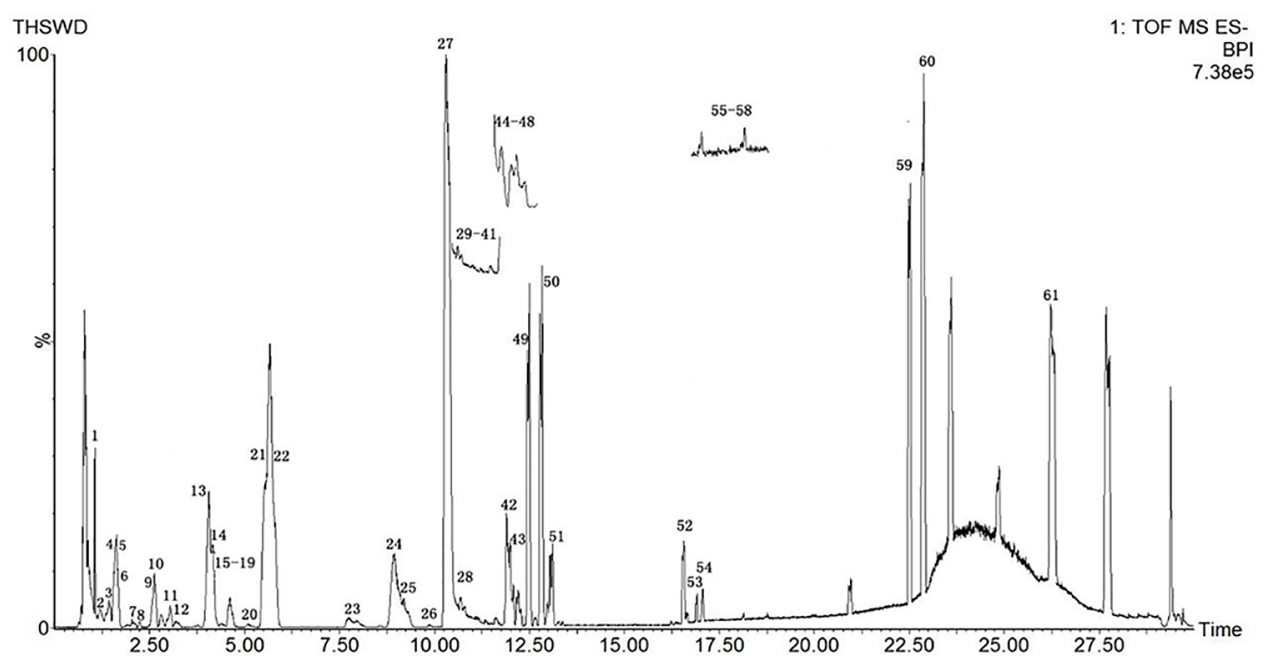

FIGURE 2 | Base peak chromatograms of THSWD in negative ion mode.

compounds that were not standard, the structure was primarily based on accurate mass and tandem MS. In this study, the formulas were based on the high-precision excimer $[\mathrm{M}+\mathrm{H}]^{+}$, $[\mathrm{M}+\mathrm{Na}]^{+},[\mathrm{M}-\mathrm{H}]^{-}$, and $[\mathrm{M}+\mathrm{HCOO}]^{-}$within a mass error of 10 ppm and a partial isotopic abundance. We then searched chemical databases such as Chemspider (www.chemspider.com) and Massbank (http://www.massbank.jp) for the most rational formulas. When all isomers were matched, the structures previously reported from the six herbs of THSWD had a higher probability than the other isomers. Finally, fragment ions were used to confirm the chemical structure further. The structures underlying the main activity in THSWD are shown in Figure 3.

\section{Phenolic Acids}

Seven phenolic acids of THSWD were identified in positive ion mode. Most from Conioselinum anthriscoides 'Chuanxiong' and
Angelica sinensis (Oliv.) Diels were ferulic acid, chlorogenic acid, caffeic acid, and vanillic acid, and gallic acid was from Paeonia lactiflora Pall. The retention time and MS information of each chemical component in THSWD were determined by UPLC-MS, and the chemical composition was confirmed by combining the extracted ion current map with data in the relevant literature. Compound 41 showed the $t_{R}$ at the 15.2-min peak obtained in ESI'mode at the $m / z 194.059$ ion peak. The literature (Lin et al., 1998; Huang and Song, 2001; Lu et al., 2004) reports that the relative molecular mass of ferulic acid in Conioselinum anthriscoides 'Chuanxiong' (syn. Ligusticum chuanxiong Hort) was 194. It was speculated that $m / z 194.059$ was its quasimolecular ion peak, and $\mathrm{MS}^{2}$ analysis was performed on $\mathrm{m} / \mathrm{z}$ 193, which were $m / z 279.0198,163.0395,92.0262$, and other fragment peaks. According to the elemental composition analysis, the molecular formula is $\mathrm{C}_{10} \mathrm{H}_{10} \mathrm{O}_{4}$. It showed a 
TABLE 1 | Compounds identified in THSWD by UPLC-Q-TOF-MS in positive ion mode.

\begin{tabular}{|c|c|c|c|c|c|c|c|}
\hline $\begin{array}{l}\text { Peak } \\
\text { No. }\end{array}$ & Indentify & $\begin{array}{l}\text { Molecular } \\
\text { formula }\end{array}$ & $\begin{array}{l}\text { Mass } \\
\text { error } \\
\text { (ppm) }\end{array}$ & $\begin{array}{c}\mathrm{RT} \\
(\mathrm{min})\end{array}$ & $\begin{array}{c}\text { Measured } \\
\text { mass }[\mathrm{M}+\mathrm{H}] \\
+/[\mathrm{M}+\mathrm{Na}]+\end{array}$ & Source $^{a}$ & MS2 \\
\hline 1 & 8-Epiloganic acid & $\mathrm{C}_{16} \mathrm{H}_{24} \mathrm{O}_{10}$ & 0.7 & 1.08 & $-/ 399.1264$ & S & $541.1764,201.0082,167.9970$ \\
\hline 2 & Guanosine & $\mathrm{C}_{10} \mathrm{H}_{13} \mathrm{~N}_{5} \mathrm{O}_{5}$ & 1.8 & 1.52 & 284.0994/- & $\mathrm{H}$ & $152.0565,135.0300$ \\
\hline 3 & Gallic acid & $\mathrm{C}_{7} \mathrm{H}_{6} \mathrm{O}_{5}$ & 0.2 & 1.6 & 171.0288/- & $\mathrm{H}, \mathrm{B}$ & $365.1068,153.0187,98.9755$ \\
\hline 4 & 1'-O-galloylsucrose & $\mathrm{C}_{19} \mathrm{H}_{26} \mathrm{O}_{15}$ & -6.2 & 1.98 & $-/ 517.1132$ & $\mathrm{~B}$ & $365.1098,261.0313,153.0174,120.0813$ \\
\hline 5 & Rehmannioside D & $\mathrm{C}_{27} \mathrm{H}_{42} \mathrm{O}_{20}$ & 3.5 & 2.01 & $-/ 709.2186$ & S & $365.1099,153.0174,120.0813$ \\
\hline 6 & $\begin{array}{l}\text { 5-Hydroxymethyl-2- } \\
\text { furfural }\end{array}$ & $\mathrm{C}_{6} \mathrm{H}_{6} \mathrm{O}_{3}$ & -1.7 & 2.24 & $127.0388 /-$ & $S, D$ & $181.9670,164.9865,109.0290$ \\
\hline 7 & indole-3-carboxaldehyde & $\mathrm{C}_{9} \mathrm{H}_{7} \mathrm{NO}$ & -3.3 & 3.38 & $146.0596 /-$ & $\mathrm{H}$ & $188.0703,146.0596,143.0722,118.0646$ \\
\hline 8 & P-Hydroxybenzoic acid & $\mathrm{C}_{7} \mathrm{H}_{6} \mathrm{O}_{3}$ & 0.1 & 3.76 & 139.039/- & $\mathrm{H}$ & $201.0096,121.0296$ \\
\hline 9 & Chlorogenic acid & $\mathrm{C}_{16} \mathrm{H}_{18} \mathrm{O}_{9}$ & 5 & 4 & $\begin{array}{l}355.1041 / \\
377.0968\end{array}$ & $\mathrm{D}$ & $211.0240,181.0132,147.0446$ \\
\hline 10 & Neo-carthamin & $\mathrm{C}_{43} \mathrm{H}_{44} \mathrm{O}_{24}$ & 1.1 & 4.02 & $451.124 /-$ & $\mathrm{H}$ & $313.0686,247.0266,211.0240,181.0132,147.0446$ \\
\hline 11 & Safflor yellow A & $\mathrm{C}_{27} \mathrm{H}_{30} \mathrm{O}_{16}$ & 5.8 & 4.03 & $611.1642 /-$ & $\mathrm{H}$ & $353.1192,201.0065,105.0334$ \\
\hline 12 & Syringin & $\mathrm{C}_{17} \mathrm{H}_{24} \mathrm{O}_{9}$ & 0.1 & 4.73 & $-/ 395.1313$ & $\mathrm{H}$ & $411.1068,395.1348,161.0590$ \\
\hline 13 & RehMapicroside & $\mathrm{C}_{16} \mathrm{H}_{26} \mathrm{O}_{8}$ & -8.1 & 4.77 & $-/ 369.149$ & $\mathrm{H}$ & $411.1068,395.1348,161.0590$ \\
\hline 14 & Amygdalin & $\mathrm{C}_{20} \mathrm{H}_{27} \mathrm{NO}_{11}$ & -0.6 & 5.63 & $\begin{array}{l}458.1581 / \\
480.1474\end{array}$ & $\mathrm{~T}$ & $496.1171,480.1473$ \\
\hline 15 & Prunasin & $\mathrm{C}_{14} \mathrm{H}_{17} \mathrm{NO}_{6}$ & 0.9 & 7.68 & $-/ 318.0951$ & $\mathrm{~T}$ & $334.0687,318.0956,201.0082$ \\
\hline 16 & 3-O-Feruloylquinic acid & $\mathrm{C}_{17} \mathrm{H}_{20} \mathrm{O}_{9}$ & 4 & 8.62 & 369.1195/- & $\mathrm{T}$ & \\
\hline 17 & paeoni-florigenone & $\mathrm{C}_{17} \mathrm{H}_{18} \mathrm{O}_{6}$ & 1.1 & 8.88 & 319.118/- & $\mathrm{B}$ & $503.1525,105.0335$ \\
\hline 18 & $\begin{array}{l}\text { 6-Hydroxyapigenin-6-O- } \\
\beta \text {-D-glucoside-7-O- } \beta \text {-D- } \\
\text { glucuronide }\end{array}$ & $\mathrm{C}_{27} \mathrm{H}_{28} \mathrm{O}_{17}$ & 2 & 10.28 & $625.1412 /-$ & $H$ & $519.1216,323.0439,133.0644$ \\
\hline 19 & Ferulaldehyde & $\mathrm{C}_{10} \mathrm{H}_{10} \mathrm{O}_{3}$ & -2.1 & 10.28 & 179.0699/- & C,D & $519.1216,503.1522,179.0697,151.0750$ \\
\hline 20 & Albiflorin & $\mathrm{C}_{23} \mathrm{H}_{28} \mathrm{O}_{11}$ & -0.1 & 10.28 & $\begin{array}{l}481.1631 / \\
503.1523\end{array}$ & $\mathrm{~B}$ & $519.1216,503.1522,500.1425,179.0697,151.0750,133.0644$ \\
\hline 21 & Paeonilactone B & $\mathrm{C}_{10} \mathrm{H}_{12} \mathrm{O}_{4}$ & -1.7 & 10.28 & $197.0805 /-$ & $\mathrm{B}$ & $519.1216,503.1522,500.1425,179.0697,151.0750,133.0644$ \\
\hline 22 & Anhydrosafflor yellow B & $\mathrm{C}_{48} \mathrm{H}_{52} \mathrm{O}_{26}$ & 6.2 & 10.29 & $1045.2885 /-$ & $\mathrm{H}$ & $519.1216,503.1522,500.1425,179.0697,151.0750$ \\
\hline 23 & Lactiflorin & $\mathrm{C}_{23} \mathrm{H}_{26} \mathrm{O}_{10}$ & -0.1 & 10.29 & 463.1598/- & $\mathrm{B}$ & $519.1216,503.1522,500.1425,179.0697,151.0750,133.0644$ \\
\hline 24 & $\begin{array}{l}\text { Quercetin- 3,7 - O - } \beta- \\
\text { D -glucoside }\end{array}$ & $\mathrm{C}_{27} \mathrm{H}_{30} \mathrm{O}_{17}$ & 0.7 & 10.54 & $\begin{array}{l}627.156 / \\
659.1488\end{array}$ & $H$ & 303.05 \\
\hline 25 & 6-Methoxycoumarin & $\mathrm{C}_{10} \mathrm{H}_{8} \mathrm{O}_{3}$ & -2.3 & 10.67 & $177.0542 /-$ & $\mathrm{D}$ & $519.1263,287.0570,177.0547,171.9834,89.0389$ \\
\hline 26 & paeoniflorin & $\mathrm{C}_{23} \mathrm{H}_{28} \mathrm{O}_{11}$ & 0.7 & 10.75 & $-/ 503.1527$ & $\mathrm{~B}$ & $503.1530,201.0068,163.0387$ \\
\hline 27 & 6-hydroxykaempferol & $\mathrm{C}_{15} \mathrm{H}_{10} \mathrm{O}_{7}$ & 2.9 & 11.29 & 303.0503/- & $\mathrm{T}, \mathrm{H}$ & $491.2935,476.3285,453.3431$ \\
\hline 28 & Cistanoside A & $\mathrm{C}_{36} \mathrm{H}_{48} \mathrm{O}_{20}$ & -1.5 & 11.34 & $-/ 823.2619$ & $\mathrm{D}$ & $693.1618,635.1593,351.1442,177.0546,145.0277$ \\
\hline 29 & Benzoic acid & $\mathrm{C}_{7} \mathrm{H}_{6} \mathrm{O}_{2}$ & 7.2 & 11.36 & $-/ 145.027$ & $\mathrm{~B}$ & $351.1442,289.0703,177.0546$ \\
\hline 30 & Galloylpaeoniflorin & $\mathrm{C}_{30} \mathrm{H}_{32} \mathrm{O}_{15}$ & 1.6 & 11.91 & $\begin{array}{l}633.1752 / \\
655.1644\end{array}$ & $\mathrm{~B}$ & $655.1640,153.0181,147.0443$ \\
\hline 31 & Saffloquinoside A & $\mathrm{C}_{27} \mathrm{H}_{30} \mathrm{O}_{15}$ & 1 & 12.04 & $\begin{array}{c}595.1663 / \\
617.159\end{array}$ & $H$ & $503.1527,287.0553,177.0544$ \\
\hline 32 & Isosafrole & $\mathrm{C}_{10} \mathrm{H}_{10} \mathrm{O}_{2}$ & -1 & 12.13 & $163.0752 /-$ & C,D & 91.0546 \\
\hline 33 & $\begin{array}{l}\text { Isorhamnetin-3-O- } \\
\text { nehesperridin }\end{array}$ & $\mathrm{C}_{28} \mathrm{H}_{32} \mathrm{O}_{16}$ & 1.2 & 12.18 & $\begin{array}{l}625.177 / \\
657.1698\end{array}$ & C & $814.5436,317.0659,302.0421,301.0351$ \\
\hline 34 & 6-hydroxyapigenin & $\mathrm{C} 8 \mathrm{H} 4 \mathrm{O} 3$ & -4.5 & 12.19 & $287.0537 /-$ & $\mathrm{D}$ & $673.4566,579.2918,363.1112,317.1154,301.1413,160.9908,149.0233$ \\
\hline 35 & Acetoside & $\mathrm{C}_{29} \mathrm{H}_{36} \mathrm{O}_{15}$ & 4.3 & 12.26 & $-/ 647.1974$ & S & $287.0555,163.0388$ \\
\hline 36 & $\begin{array}{l}\text { Kaempferol 3-O- } \\
\text { rutinoside }\end{array}$ & $\mathrm{C}_{27} \mathrm{H}_{30} \mathrm{O}_{15}$ & 6.5 & 12.4 & $595.1696 /-$ & $\mathrm{T}, \mathrm{H}$ & $519.1306,503.1549,317.0680,302.0432,205.0721,181.0127,140.0510$ \\
\hline 37 & cosmosiin & $\mathrm{C}_{21} \mathrm{H}_{20} \mathrm{O}_{10}$ & 2.6 & 12.4 & 433.114/- & C & $301.1412,149.0232$ \\
\hline 38 & Carthamidin & $\mathrm{C}_{15} \mathrm{H}_{12} \mathrm{O}_{6}$ & 0.6 & 12.59 & 289.0708/- & $H$ & $153.0181,147.0443$ \\
\hline 39 & Senkyunolide H & $\mathrm{C}_{12} \mathrm{H}_{16} \mathrm{O}_{4}$ & -1.8 & 13.13 & $-/ 247.0936$ & C & $1165.1875,1045.4538,926.4874,789.3951,712.7139,601.8298$ \\
\hline 40 & $\begin{array}{l}\text { 4-hydroxy-3- } \\
\text { butylphthalide }\end{array}$ & $\mathrm{C}_{12} \mathrm{H}_{14} \mathrm{O}_{3}$ & -0.7 & 13.15 & 207.1014/- & C,D & $247.0952,147.0446,91.0545$ \\
\hline 41 & ferulic acid & $\mathrm{C}_{10} \mathrm{H}_{10} \mathrm{O}_{4}$ & 4.8 & 15.2 & $-/ 217.0482$ & C,D & $279.0198,163.0395,92.0262$ \\
\hline 42 & safflospermidine A & $\mathrm{C}_{34} \mathrm{H}_{37} \mathrm{~N}_{3} \mathrm{O}_{6}$ & 1.8 & 15.81 & $\begin{array}{l}584.2766 / \\
606.2693\end{array}$ & $\mathrm{H}$ & $204.1032,147.0438$ \\
\hline 43 & oxybenzoyl-paeoniflorin & $\mathrm{C}_{30} \mathrm{H}_{32} \mathrm{O}_{12}$ & 1.3 & 16.55 & $-/ 607.1794$ & $\mathrm{~B}$ & $607.1794,249.0760,105.0335$ \\
\hline 44 & Benzoylpaeoniflorin & $\mathrm{C} 30 \mathrm{H} 32 \mathrm{O} 12$ & 1.1 & 16.65 & 585.1973/- & $\mathrm{B}$ & 133.0663 \\
\hline 45 & Jionoside B1 & $\mathrm{C}_{37} \mathrm{H}_{50} \mathrm{O}_{20}$ & -5.9 & 17.09 & $-/ 837.2738$ & S & $228.2324,107.0853,105.0695$ \\
\hline 46 & Z-Butylidenephthalide & $\mathrm{C}_{12} \mathrm{H}_{12} \mathrm{O}_{2}$ & -0.8 & 17.15 & $189.0908 /-$ & C,D & $608.1815,219.0692,128.0629$ \\
\hline 47 & Ligustilide & $\mathrm{C}_{12} \mathrm{H}_{14} \mathrm{O}_{2}$ & -2.9 & 17.22 & 191.1061/- & C,D & $670.3756,447.2399,355.2629,181.1199,165.0908,92.0252$ \\
\hline
\end{tabular}


TABLE 1 | Continued

\begin{tabular}{|c|c|c|c|c|c|c|c|}
\hline $\begin{array}{c}\text { Peak } \\
\text { No. }\end{array}$ & Indentify & $\begin{array}{l}\text { Molecular } \\
\text { formula }\end{array}$ & $\begin{array}{l}\text { Mass } \\
\text { error } \\
\text { (ppm) }\end{array}$ & $\begin{array}{c}\mathrm{RT} \\
(\mathrm{min})\end{array}$ & $\begin{array}{c}\text { Measured } \\
\text { mass }[\mathrm{M}+\mathrm{H}] \\
+/[\mathrm{M}+\mathrm{Na}]+\end{array}$ & Source $^{a}$ & MS2 \\
\hline 48 & $\begin{array}{l}\text { 4,5-dihydro-3- } \\
\text { butylphthalide }\end{array}$ & $\mathrm{C}_{12} \mathrm{H}_{16} \mathrm{O}_{2}$ & 4 & 18.27 & 193.1231/- & C & 242.2845 \\
\hline 49 & 3-Butylphthalide & $\mathrm{C}_{12} \mathrm{H}_{14} \mathrm{O}_{2}$ & 1.1 & 19.12 & 191.1069/- & C,D & $191.1081,128.0624,115.0542$ \\
\hline 50 & Levistolide A & $\mathrm{C}_{24} \mathrm{H}_{28} \mathrm{O}_{4}$ & 0.6 & 20.62 & $\begin{array}{c}381.2063 / \\
403.199\end{array}$ & D & $419.1643,311.2948,256.2643$ \\
\hline 51 & Dibutyl phthalate & $\mathrm{C}_{16} \mathrm{H}_{22} \mathrm{O}_{4}$ & 2.7 & 20.75 & $-/ 301.1418$ & C & $787.52097,743.4928,337.3110$ \\
\hline 52 & $\begin{array}{l}\text { 3-Butylidene-7- } \\
\text { hydroxyphthalide }\end{array}$ & $\mathrm{C}_{12} \mathrm{H}_{12} \mathrm{O}_{3}$ & 0.3 & 20.77 & $\begin{array}{l}205.086 / \\
227.0787\end{array}$ & C & $301.1412,149.0232$ \\
\hline 53 & Z-6,8',7,3'- diligustilide & $\mathrm{C}_{24} \mathrm{H}_{28} \mathrm{O}_{4}$ & -0.6 & 20.82 & $381.2058 /-$ & C & $510.3556,492.3458,473.2644,327.2904,191.1072$ \\
\hline 54 & Eugeniin & $\mathrm{C}_{41} \mathrm{H}_{30} \mathrm{O}_{26}$ & 6.5 & 22.3 & $-/ 961.098$ & $\mathrm{H}$ & $283.2633,281.2476$ \\
\hline 55 & Daucosterol & $\mathrm{C}_{35} \mathrm{H}_{60} \mathrm{O}_{6}$ & -9.4 & 23.27 & $-/ 599.4226$ & $\mathrm{H}, \mathrm{C}, \mathrm{D}$ & $283.2632,243.1214,95.0858$ \\
\hline 56 & Ligustrazin & $\mathrm{C}_{8} \mathrm{H}_{12} \mathrm{~N}_{2}$ & -1.9 & 26.77 & 137.1071/- & $\mathrm{C}$ & $302.0432,205.0720,181.0127$ \\
\hline
\end{tabular}

${ }^{a} H, T, D, C, B$, S indicate Prunus persica (L.) Batsch, Carthamus tinctorius L., Angelica sinensis (Oliv.) Diels, Conioselinum anthriscoides 'Chuanxiong' (syn. Ligusticum chuanxiong Hort), Paeoniae lactiflora Pall., and Rehmannia glutinosa (Gaertn.) DC, respectively.

TABLE 2 | Compounds identified in THSWD by UPLC-Q-TOF-MS in negative ion mode.

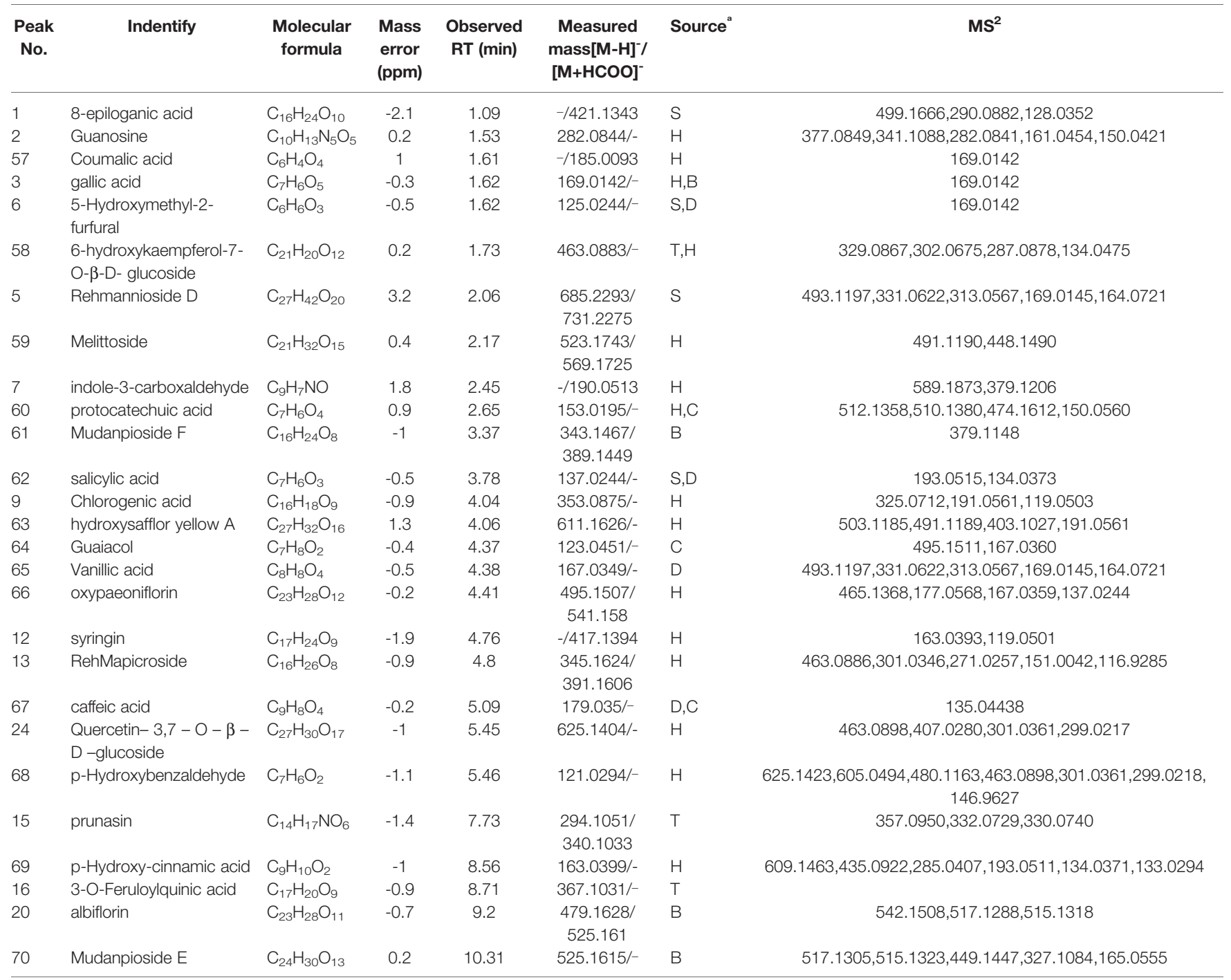


TABLE 2 | Continued

\begin{tabular}{|c|c|c|c|c|c|c|c|}
\hline $\begin{array}{l}\text { Peak } \\
\text { No. }\end{array}$ & Indentify & $\begin{array}{l}\text { Molecular } \\
\text { formula }\end{array}$ & $\begin{array}{l}\text { Mass } \\
\text { error } \\
\text { (ppm) }\end{array}$ & $\begin{array}{l}\text { Observed } \\
\text { RT (min) }\end{array}$ & $\begin{array}{c}\text { Measured } \\
\text { mass }[\mathrm{M}-\mathrm{H}]^{-/} \\
{[\mathrm{M}+\mathrm{HCOO}]^{-}}\end{array}$ & Source $^{a}$ & $\mathbf{M S}^{2}$ \\
\hline 18 & $\begin{array}{l}\text { 6-hydroxyapigenin-6-O- } \\
\beta \text {-D-glucoside-7-O- } \beta \text {-D- } \\
\text { glucuronide }\end{array}$ & $\mathrm{C}_{27} \mathrm{H}_{28} \mathrm{O}_{17}$ & -0.6 & 10.43 & $623.1268 /-$ & $H$ & $525.1612,517.1305,515.1323,449.1447,327.1084,121.0294$ \\
\hline 71 & $\begin{array}{l}\text { 6-hydroxyapigenin-3,6- } \\
\text { di }-O-\beta-D-\text { glucoside }\end{array}$ & $\mathrm{C}_{27} \mathrm{H}_{30} \mathrm{O}_{17}$ & 1.2 & 10.56 & $625.1417 /-$ & $H$ & $\begin{array}{c}625.1414,609.1461,463.0871,462.0796,301.0347,271.0248,243 . \\
0307,139.0042\end{array}$ \\
\hline 41 & Ferulic acid & $\mathrm{C}_{10} \mathrm{H}_{10} \mathrm{O}_{4}$ & 0.1 & 10.69 & 193.0507/- & $H, C, D$ & $193.0511,134.0370,133.0294,132.0210$ \\
\hline 72 & Purpureaside C & $\mathrm{C}_{35} \mathrm{H}_{46} \mathrm{O}_{20}$ & 2.9 & 10.78 & $785.2533 /-$ & S & $623.2193,495.1525,193.0507,161.0244,137.0246$ \\
\hline 73 & safflor yellow & $\mathrm{C}_{27} \mathrm{H}_{30} \mathrm{O}_{15}$ & 0.9 & 11.05 & 609.1466/- & $\mathrm{H}$ & $301.0350,300.0277$ \\
\hline 74 & ISOQUERCITRIN & $\mathrm{C}_{21} \mathrm{H}_{20} \mathrm{O}_{12}$ & -0.8 & 11.14 & 463.0878/- & $\mathrm{T}, \mathrm{H}$ & $271.0248,243.0299,116.9286$ \\
\hline 75 & $\begin{array}{l}\text { kaempferol 3-O- } \beta \text { - } \\
\text { sophoroside }\end{array}$ & $\mathrm{C}_{27} \mathrm{H}_{30} \mathrm{O}_{16}$ & 0.1 & 11.21 & $609.1462 /-$ & $\mathrm{T}, \mathrm{H}$ & $609.1462,284.0325,227.0352,151.0040$ \\
\hline 76 & $\begin{array}{l}\text { Dihydrophaseic acid-4'- } \\
\text { O- } \beta \text {-D-glucoside methyl } \\
\text { ester }\end{array}$ & $\mathrm{C}_{22} \mathrm{H}_{34} \mathrm{O}_{10}$ & -0.8 & 11.25 & $-/ 503.213$ & $H$ & $610.1502,493.1855,430.0922,361.1669,283.0260,145.0286$ \\
\hline 28 & Cistanoside A & $\mathrm{C}_{36} \mathrm{H}_{48} \mathrm{O}_{20}$ & 3.3 & 11.34 & $\begin{array}{l}799.2692 / \\
845.2765\end{array}$ & $\mathrm{D}$ & $799.2694,624.2226,623.2197,193.0509,175.0399,161.0245$ \\
\hline 11 & safflor yellow A & $\mathrm{C}_{27} \mathrm{H}_{30} \mathrm{O}_{16}$ & -1.5 & 11.38 & $\begin{array}{l}611.1608 / \\
657.1681\end{array}$ & $\mathrm{H}$ & $609.1464,301.0351,300.0277$ \\
\hline 77 & Rehmaionoside B & $\mathrm{C}_{19} \mathrm{H}_{34} \mathrm{O}_{8}$ & -1.4 & 11.83 & $-/ 435.223$ & S & $695.5073,595.1719,425.1937,389.0877,347.0778,149.0247$ \\
\hline 39 & Senkyunolide H & $\mathrm{C}_{12} \mathrm{H}_{16} \mathrm{O}_{4}$ & 0.7 & 11.91 & $223.0977 /-$ & C,D & $631.1673,169.0140,161.0242$ \\
\hline 30 & galloylpaeoniflorin & $\mathrm{C}_{30} \mathrm{H}_{32} \mathrm{O}_{15}$ & 0.9 & 11.92 & $631.1674 /-$ & $\mathrm{B}$ & $\begin{array}{c}631.1673,613.1566,399.0927,313.0564,271.0464,169.0140 \\
161.0242\end{array}$ \\
\hline 10 & neo-carthamin & $\mathrm{C}_{43} \mathrm{H}_{44} \mathrm{O}_{24}$ & -1.1 & 11.93 & $449.1084 /-$ & $\mathrm{H}$ & $631.1673,313.0564,271.0464,169.0140,161.0242$ \\
\hline 36 & $\begin{array}{l}\text { Kaempferol 3-O- } \\
\text { rutinoside }\end{array}$ & $\mathrm{C}_{27} \mathrm{H}_{30} \mathrm{O}_{15}$ & 0.7 & 12.05 & $593.1516 /-$ & $\mathrm{T}, \mathrm{H}$ & $\begin{array}{c}593.1513,285.0399,284.0327,255.0230,227 \\
0348,175.0399\end{array}$ \\
\hline 45 & Jionoside B1 & $\mathrm{C}_{37} \mathrm{H}_{50} \mathrm{O}_{20}$ & 2.6 & 12.08 & $\begin{array}{l}813.2844 / \\
859.2916\end{array}$ & S & $593.1513,285.0399,284.0327,255.0230,227.0348,175.0399$ \\
\hline 78 & p-Hydroxyacetophenone & $\mathrm{C}_{8} \mathrm{H}_{8} \mathrm{O}_{2}$ & -1.2 & 12.13 & $135.045 /-$ & $H$ & $801.1863,637.1470,467.2142,146.9655$ \\
\hline 37 & cosmosiin & $\mathrm{C}_{21} \mathrm{H}_{20} \mathrm{O}_{10}$ & -1.8 & 12.14 & $431.0976 /-$ & $H$ & $801.1863,626.1655,146.9655$ \\
\hline 33 & $\begin{array}{l}\text { Isorhamnetin-3-O- } \\
\text { nehesperridin }\end{array}$ & $\mathrm{C}_{28} \mathrm{H}_{32} \mathrm{O}_{16}$ & 1.6 & 12.19 & 623.1627/- & $\mathrm{C}$ & $623.1624,315.0504,243.0300$ \\
\hline 79 & $\begin{array}{l}\text { kaeMpferol 3-O-D- } \\
\text { glucopyranoside }\end{array}$ & $\mathrm{C}_{21} \mathrm{H}_{20} \mathrm{O}_{11}$ & -1 & 12.27 & $447.0928 /-$ & $\mathrm{T}, \mathrm{H}$ & $284.0330,227.0351,161.0242,133.0295$ \\
\hline 80 & Isoacteoside & $\mathrm{C}_{29} \mathrm{H}_{36} \mathrm{O}_{15}$ & -0.2 & 12.27 & 623.1985/- & $\mathrm{T}$ & $\begin{array}{c}574.1599,424.1033,364.0819,311.0931,161.0238 \\
133.0197\end{array}$ \\
\hline 81 & Sophoricoside & $\mathrm{C}_{21} \mathrm{H}_{20} \mathrm{O}_{10}$ & -0.5 & 12.41 & $\begin{array}{l}431.1054 / \\
477.1036\end{array}$ & $\mathrm{H}$ & $611.1398,403.0806,287.0566,162.8394,119.0501$ \\
\hline 82 & Jionoside A & $\mathrm{C}_{30} \mathrm{H}_{38} \mathrm{O}_{15}$ & 0 & 13.12 & 637.2138/- & $\mathrm{D}$ & $451.3276,347.0761,174.9555,161.0247$ \\
\hline 23 & lactiflorin & $\mathrm{C}_{23} \mathrm{H}_{26} \mathrm{O}_{10}$ & -0.2 & 13.26 & $\begin{array}{l}461.1525 / \\
507.1507\end{array}$ & $\mathrm{~B}$ & $539.1899,497.1225,237.0770,121.0299$ \\
\hline 44 & benzoylpaeoniflorin & $\mathrm{C}_{30} \mathrm{H}_{32} \mathrm{O}_{12}$ & 1.8 & 16.55 & $\begin{array}{c}583.1905 / \\
629.1887\end{array}$ & $\mathrm{~B}$ & $619.1593,121.0294$ \\
\hline 43 & oxybenzoyl-paeoniflorin & $\mathrm{C}_{30} \mathrm{H}_{32} \mathrm{O}_{12}$ & 0.7 & 16.82 & $\begin{array}{c}583.19898 / \\
629.188\end{array}$ & $\mathrm{~B}$ & $134.0371,121.0294$ \\
\hline 83 & $\begin{array}{l}\text { 4,7-dihydroxy-3- } \\
\text { butylphthalide }\end{array}$ & $\mathrm{C}_{12} \mathrm{H}_{14} \mathrm{O}_{4}$ & 0.6 & 17.17 & $221.0821 /-$ & $\mathrm{C}$ & 290.0673,205.0884,161.0975 \\
\hline 84 & Senkyunolide G & $\mathrm{C}_{12} \mathrm{H}_{16} \mathrm{O}_{3}$ & -0.6 & 17.24 & 207.1025/- & C & $265.0631,221.8433,163.1134$ \\
\hline 40 & $\begin{array}{l}\text { 4-hydroxy-3- } \\
\text { butylphthalide }\end{array}$ & $\mathrm{C}_{12} \mathrm{H}_{14} \mathrm{O}_{3}$ & -0.8 & 17.38 & 205.0869/- & $\mathrm{C}$ & $237.0929,134.0374$ \\
\hline 85 & senkyunolide E & $\mathrm{C}_{12} \mathrm{H}_{12} \mathrm{O}_{3}$ & -0.5 & 17.73 & 203.0713/- & $\mathrm{C}$ & $203.07211,145.0288,132.0230$ \\
\hline 48 & $\begin{array}{l}\text { 4,5-dihydro-3- } \\
\text { butylphthalide }\end{array}$ & $\mathrm{C}_{12} \mathrm{H}_{16} \mathrm{O}_{2}$ & -4.7 & 17.91 & 191.1069/- & $\mathrm{C}$ & $925.4829,701.4286,447.1355$ \\
\hline 86 & Myristic acid & $\mathrm{C}_{14} \mathrm{H}_{28} \mathrm{O}_{2}$ & -0.2 & 22.43 & 227.2016/- & $\mathrm{H}$ & $285.1609,227.2017$ \\
\hline 87 & monopalmitin & $\mathrm{C}_{19} \mathrm{H}_{38} \mathrm{O}_{4}$ & -1.6 & 22.97 & $-/ 375.2746$ & $\mathrm{H}, \mathrm{C}$ & $429.1942,99.9258$ \\
\hline 56 & Daucosterol & $\mathrm{C}_{35} \mathrm{H}_{60} \mathrm{O}_{6}$ & 0.2 & 26.31 & $-/ 621.4373$ & S & $255.2330,96.9601$ \\
\hline
\end{tabular}

${ }^{a} H, T, D, C, B$, S indicate Prunus persica (L.) Batsch, Carthamus tinctorius L., Angelica sinensis (Oliv.) Diels, Conioselinum anthriscoides 'Chuanxiong' (syn. Ligusticum chuanxiong Hort), Paeoniae lactiflora Pall., and Rehmannia glutinosa (Gaertn.) DC, respectively. 


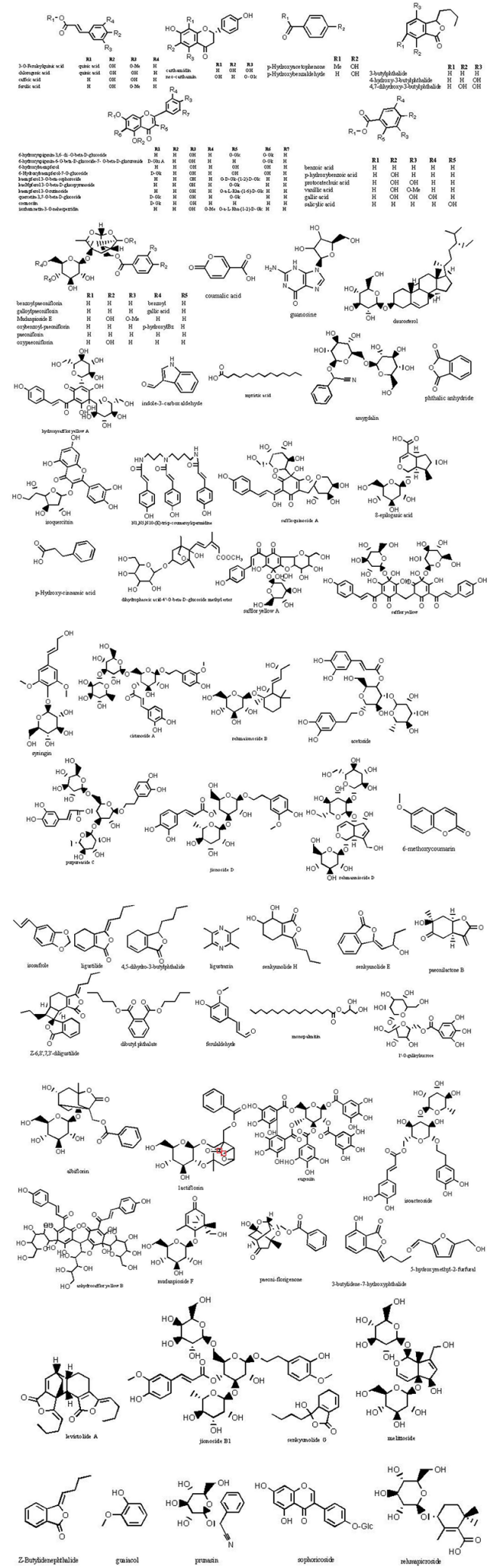

FIGURE 3 | Chemical structures of the main constitutions found in THSWD. theoretical value of the relative molecular mass of 193.0506 and a measured value of 194.059. This peak adds $\mathrm{Na}$ in ESI+ mode to obtain the ion peak with $m / z 217$. MS ${ }^{2}$ analysis was performed on $\mathrm{m} / z 217$ to obtain fragment peaks such as $\mathrm{m} / z 123$ and 151 . According to the elemental composition analysis, the molecular formula was $\mathrm{C}_{10} \mathrm{H}_{10} \mathrm{O}_{4}$, and the theoretical value of the relative molecular mass was 217.0471. The actual value was 217.0475 . Based on this, the compound was assumed to be ferulic acid.

Compound 9 showed a $[\mathrm{M}+\mathrm{H}]{ }^{+}$ion at $m / z$ 355.1041, and more abundant ions in the $\mathrm{MS}^{2}$ spectrum were $\mathrm{m} / z$ 211.0240, 181.0132, and 147.0446. It was identified as chlorogenic acid from Angelica sinensis (Oliv.) Diels. The $\mathrm{MS}^{2}$ spectrum and possible fragmentation pathways of chlorogenic acid are depicted in Figure 4. Compound 67 had a retention time of $5.09 \mathrm{~min}$, and the characteristic ion fragment is $179[\mathrm{M}-\mathrm{H}]^{-}$. It can be deduced that the molecular formula may be $\mathrm{C}_{9} \mathrm{H}_{8} \mathrm{O}_{4}$. In the further MS cleavage process, its quasi-molecular ion removes 1 molecule of $\mathrm{CO}_{2}$ to form a characteristic fragment ion $\mathrm{m} / z 135.04438$ [M-H$\mathrm{CO} 2]-$. By comparison with the standard spectrum, it was confirmed to be caffeic acid.

In negative ion mode, the retention time of compound 3 was $1.62 \mathrm{~min}$, and the peak of its excimer ion was $\mathrm{m} / z$ $169.0142[\mathrm{M}-\mathrm{H}]^{-}$. It can be deduced that the molecular formula may be $\mathrm{C}_{7} \mathrm{H}_{6} \mathrm{O}_{5}$; the error is 0.3 . In positive ion mode, the excimer ion peak was $m / z 171.0227[\mathrm{M}+\mathrm{H}]^{+}$ with an error of 0.2 . In the subsequent MS cleavage process, the quasi-molecular ion removed one molecule of $\mathrm{CO}_{2}$ to form the characteristic fragment ion $m / z 125.0215\left[\mathrm{M}-\mathrm{H}-\mathrm{CO}_{2}\right]^{-}$, indicating that the molecular structure may contain carboxylic acid groups. The related literature (Sun et al., 2009; Jiang et al., 2011; Liu et al., 2017) is consistent with the main characteristics of gallic acid, so that was inferred to be the identity of compound 3 .

\section{Flavones}

Most of the six drugs of THSWD contain flavonoids that have a high response in positive and negative ion modes, but some compounds only respond in negative ion mode with $[\mathrm{M}-\mathrm{H}]^{-}$and [M+HCOO]-. To facilitate the discussion of MS cleavage of different flavonoids in THSWD, the nomenclature of fragment ions for flavonoids was used (Ma et al., 1997; Feng et al., 2017). The main active ingredient in Carthamus tinctorius L. is safflor yellow. Viewing the information on the mass spectrum of compound 63, it showed a molecular weight at 612.1698. The fragment $m / z 611[\mathrm{M}-\mathrm{H}]^{-}$was the excimer ion peak. By reading the literature (Ning et al., 2012; Zhang et al., 2014), it was inferred to be hydroxy safflor yellow $\mathrm{A}$ with a molecular formula of $\mathrm{C}_{27} \mathrm{H}_{32} \mathrm{O}_{16}$. At the same time, compound 11 was identified as safflor yellow A. Compound 10 was identified as neocarthamin.

Some flavonoids are mostly present in the form of flavonoid glycosides. By comparison with the reference substance, it was determined that compounds $24,35,19$, and 12 were quercetin, apigenin, kaempferol, and isorhamnetin, respectively. The retention time and lysis of the reference substance were in accordance with these inferences. 


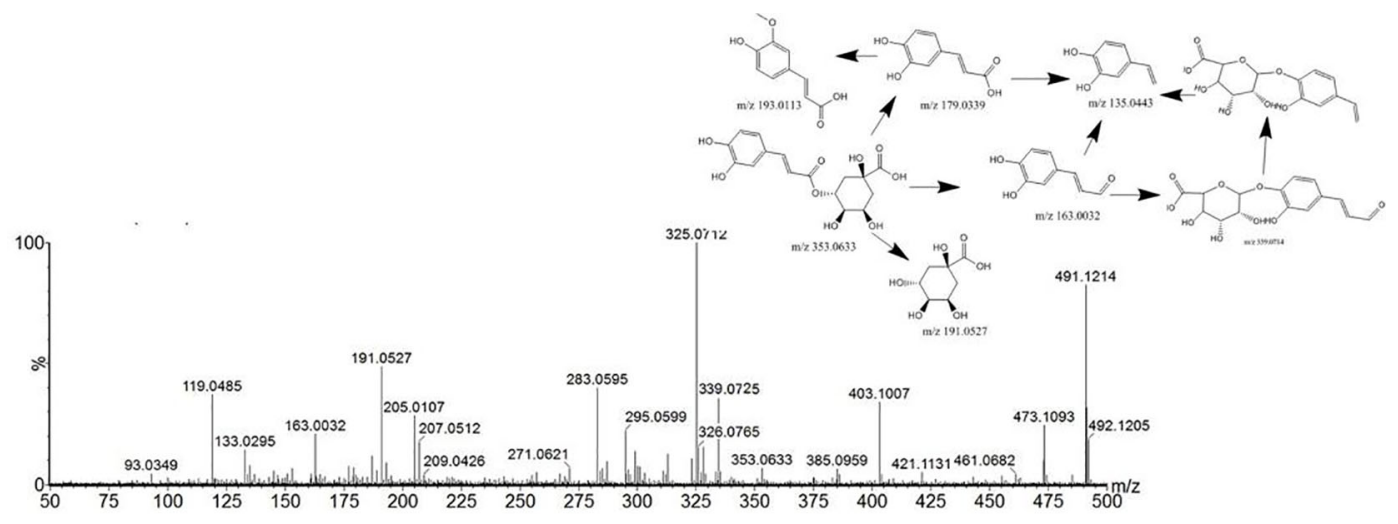

FIGURE 4 | Tandem mass spectra and possible fragment pathways of chlorogenic acid in positive ion mode.

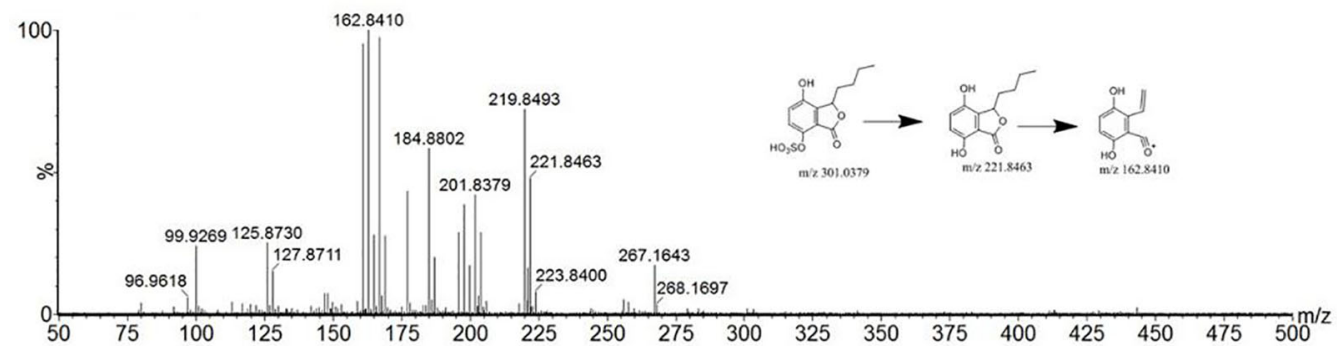

FIGURE $\mathbf{5}$ | Tandem mass spectra and possible fragment pathways of 4-hydroxy-3-butyl-benzoquinone in positive ion mode.

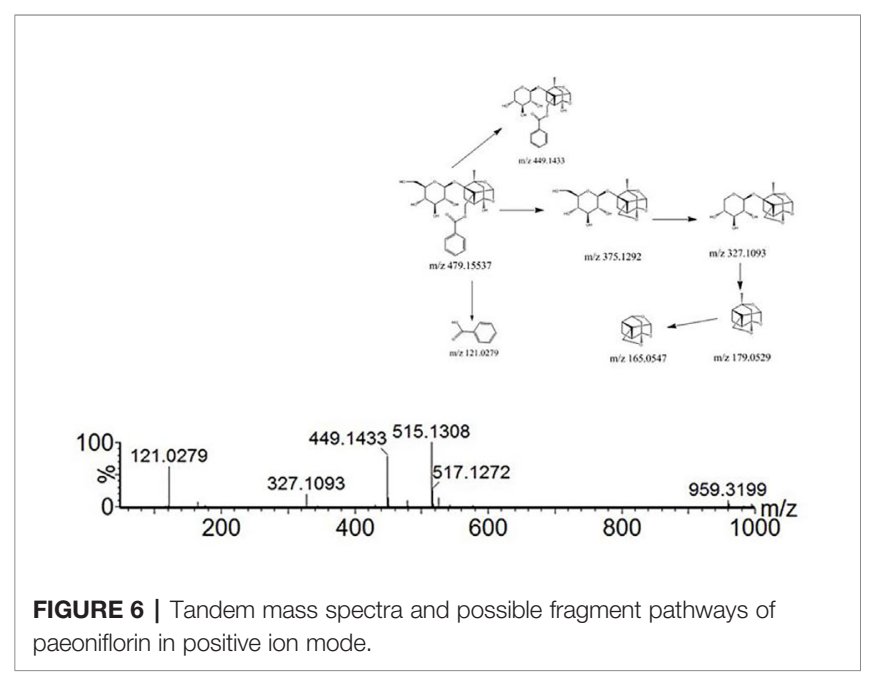

\section{Polysaccharides}

Polysaccharides are one of the main active ingredients of THSWD and are mainly from Angelica sinensis (Oliv.) Diels and Rehmannia glutinosa (Gaertn.) DC. Compound 4 had a retention time of $1.98 \mathrm{~min}$, the excimer ion peak was $\mathrm{m} / \mathrm{z} 493$
$[\mathrm{M}-\mathrm{H}]^{-}$, the fragment $\mathrm{m} / \mathrm{z} 331$ was formed as an excimer ion peak, and a glucose residue (162 u) was formed. The characteristic fragment was $\mathrm{m} / \mathrm{z} 169$. The ions were derived from galloyl anions, and fragment ions of $\mathrm{m} / z 125$ were generated after the gallic acyl anion underwent hydroxyl alpha cleavage to lose one molecule of $\mathrm{CO}_{2}(44 \mathrm{u})$. According to the literature, the three isomers 1'-O-galloyl sucrose, 6'-O-galloyl sucrose, and 6-O-galloyl sucrose exist in the white peony, but from the total ion chromatogram A $\mathrm{m} / \mathrm{z} 493$ was extracted, and only one peak appeared at $4.549 \mathrm{~min}$. Therefore, it is presumed that compound 4 is galloyl sucrose, but the linking position of its sugar needs to be confirmed.

\section{Volatile Compounds}

The major volatile oils were from Angelica sinensis (Oliv.) Diels and Conioselinum anthriscoides 'Chuanxiong' (syn. Ligusticum chuanxiong Hort). Compound 47 showed an $[\mathrm{M}+\mathrm{H}]^{+}$ion at $\mathrm{m} / \mathrm{z}$ 191.1061. The literature (Wang and Han, 2011) has reported that the relative molecular mass of ligustilide in C. anthriscoides 'Chuanxiong' was 190. It was speculated that $\mathrm{m} / \mathrm{z} 191$ was its quasi-molecular ion peak. $\mathrm{MS}^{2}$ analysis was performed on $\mathrm{m} / \mathrm{z}$ 191, which yielded fragment peaks such as $\mathrm{m} / \mathrm{z} 670,447$, and 355 . It showed a retention time of 17.22 in positive ion mode. According to the elemental composition analysis, the 

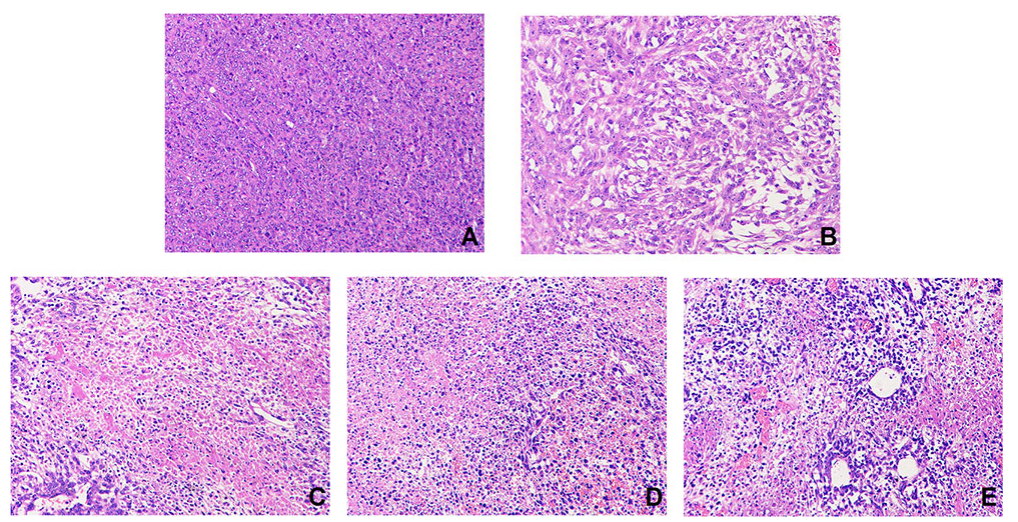

FIGURE 7 | Effect of THSWD on tumor cells in mice. Sections were stained with H\&E. Magnification, $\times 200$; scale bars, $50 \mu \mathrm{m}$. Model group (A), cisplatin group (B), THSWD H group (C), THSWD M group (D), THSWD L group (E).

TABLE 3 | IL-4, IL-10, IL-13, and TGF- $\beta 1$ expression.

\begin{tabular}{|c|c|c|c|c|}
\hline Group & IL-4(pg/ml) & IL-10(pg/ml) & TNF-a & $\begin{array}{l}\text { TGF- } \beta 1 \\
\text { (ng/ml) }\end{array}$ \\
\hline $\mathrm{N}$ & $366.21 \pm 38.07$ & $1097.42 \pm 106.03$ & $1154.64 \pm 174.62$ & $402.80 \pm 31.10$ \\
\hline$M$ & $\begin{array}{c}336.18 \\
\pm 51.74^{\# \#}\end{array}$ & $1028.10 \pm 43.31^{\#}$ & $\begin{array}{c}1657.05 \\
\pm 268.54^{\# \#}\end{array}$ & $\begin{array}{c}445.98 \\
\pm 26.76^{\#}\end{array}$ \\
\hline C & $506.78 \pm 64.39^{\star \star}$ & $\begin{array}{c}1121.88 \\
\pm 105.69^{*}\end{array}$ & $1338.12 \pm 56.04^{*}$ & $\begin{array}{c}413.13 \\
\pm 11.27^{\star}\end{array}$ \\
\hline $\mathrm{H}$ & $354.10 \pm 44.88$ & $\begin{array}{c}1112.76 \\
\pm 102.59^{* *}\end{array}$ & $1336.89+344.15^{\star}$ & $436.98 \pm 34.06$ \\
\hline$M$ & $386.41 \pm 45.83^{\star \star}$ & $\begin{array}{c}1175.52 \\
\pm 115.75^{\star}\end{array}$ & $1607.49 \pm 170.73$ & $\begin{array}{l}400.86 \\
\pm 40.15^{\star}\end{array}$ \\
\hline $\mathrm{L}$ & $343.69 \pm 40.53$ & $1048.27 \pm 143.15$ & $1335.96 \pm 296.74^{*}$ & $424.23 \pm 13.06$ \\
\hline
\end{tabular}

molecular formula was $\mathrm{C}_{12} \mathrm{H}_{14} \mathrm{O}_{2}$, the theoretical value of the relative molecular mass was 191.1061, and the measured value was 191.1061. Based on this, it was assumed that the compound was ligustilide.

Compound 40 showed a retention time of $13.15 \mathrm{~min}$ in positive ion mode, with an $[\mathrm{M}+\mathrm{H}]+$ ion at $\mathrm{m} / z$ 207.1014. The peak with $t_{R}$ of 17.38 min yielded an $m / z 205.0869$ ion peak in the ESI'. The literature (Yang et al., 2007) reports that the relative molecular mass of 4-hydroxy-3-butyl- benzoquinone in $C$. anthriscoides 'Chuanxiong' is 206. According to the $\mathrm{MS}^{2}$ results, it was assumed that the compound was 4-hydroxy-3butyl-benzoquinone. The $\mathrm{MS}^{2}$ spectrum and possible fragmentation pathways are depicted in Figure 5.

\section{Monoterpene Glycosides}

Compound 21 showed a retention time of $10.28 \mathrm{~min}$. The fragment ions in the mass spectrum were $m / z 519.1216$, $503.1522,500.1425,179.0697,151.0750$, and 133.0644, and the excimer ion peak was $m / z 479[\mathrm{M}-\mathrm{H}]^{-}$. The MS2 spectrum and possible fragmentation pathways are depicted in Figure 6. Fragment ion $\mathrm{m} / \mathrm{z} 525$ formed for the molecular ion peak plus

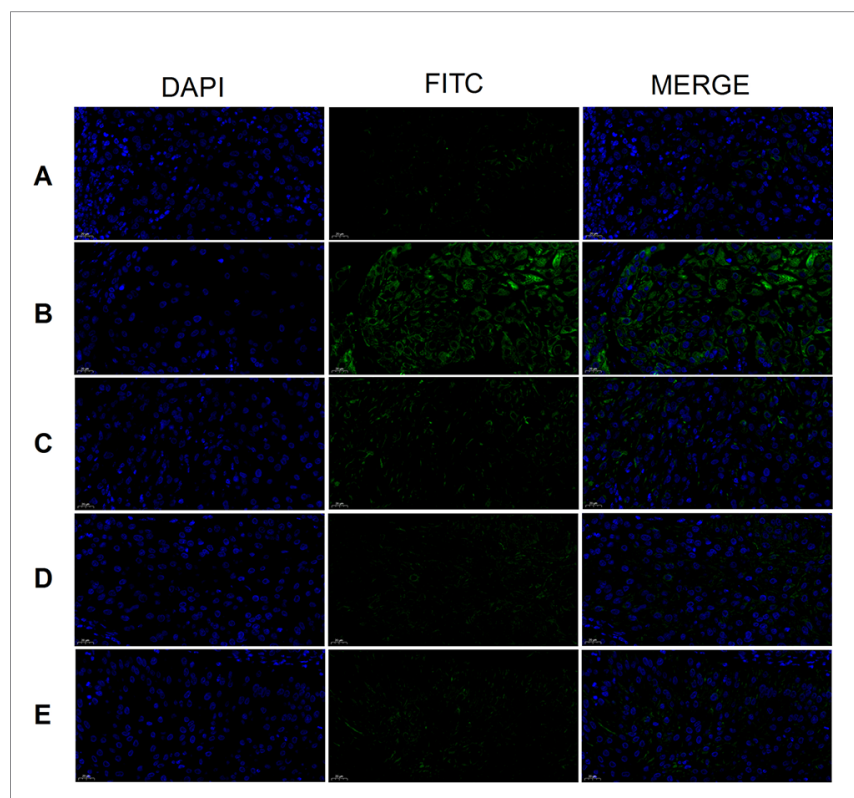

FIGURE 8 | CD44 expression (×400). Model group (A), cisplatin group (B), THSWD H group (C), THSWD M group (D), THSWD L group (E)

one molecule of formic acid (46 u). Due to the presence of a lactone ring in the structure of paeoniflorin, its acyloxy groups can be broken on both sides, losing one molecule of $\mathrm{CO}_{2}(44 \mathrm{u})$ at $\mathrm{m} / \mathrm{z} 435$. The fragment $m / z 357$ was formed as the excimer peak and lost one molecule of benzoic acid (122 u), and $\mathrm{m} / z 121$ was the missing one of the benzoic acid fragment ions. A combination of fragment $\mathrm{m} / \mathrm{z} 481[\mathrm{M}+\mathrm{H}]^{+}$and fragment $\mathrm{m} / \mathrm{z}$ $503.1522[\mathrm{M}+\mathrm{Na}]^{+}$appeared in positive ion mode, indicating that compound 21 is paeonolide $\mathrm{B}$.

For compound 26, the retention time was $12.393 \mathrm{~min}$. The fragment ions in the mass spectrum had $m / z 525,449,327$, and 165. The molecular ion peak was $m / z 479[\mathrm{M}+\mathrm{H}]^{-}$, and the 


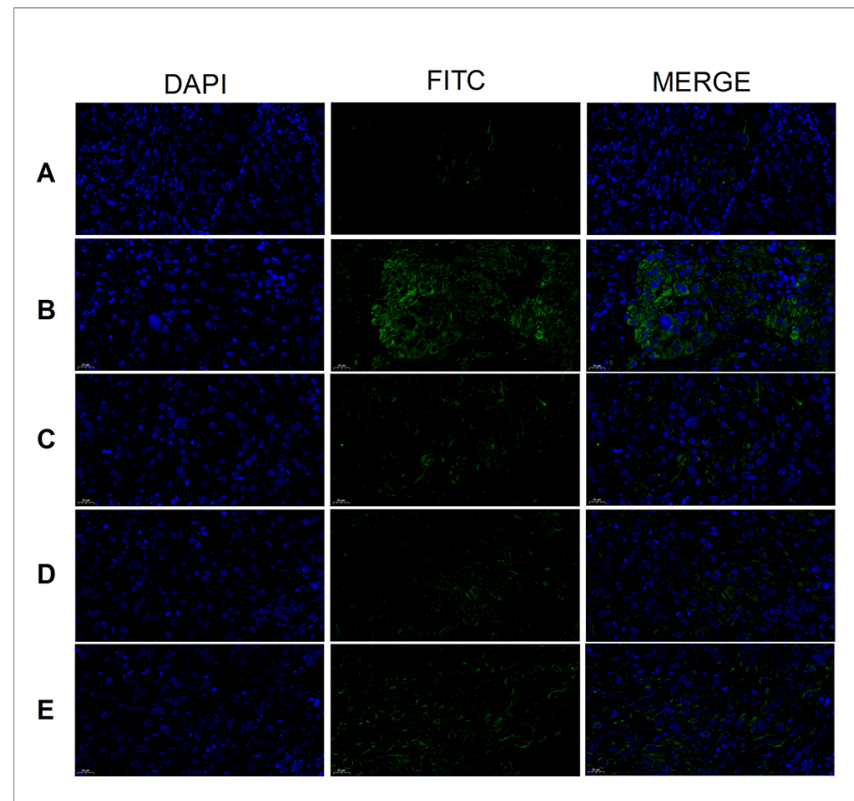

FIGURE 9 | CD117 expression (×400). Model group (A), cisplatin group (B), THSWD H group (C), THSWD M group (D), THSWD L group (E). fragment ion $m / z 525$ was the molecular ion peak plus a molecule of formic acid ( $46 \mathrm{u}) . \mathrm{m} / z 449$ is the molecular ion peak $(\mathrm{m} / z 479$ $[\mathrm{MH}]-)$ that loses a molecule of formaldehyde $(30 \mathrm{u})$ on the 6carbon residue of the glucosyl group, while the $m / z 449$ fragment ion further loses a molecule of benzoic acid (122 u) to form the $\mathrm{m} / \mathrm{z} 327$ fragment ion. $\mathrm{m} / z 165$ is a fragment of the decyl alkyl skeleton structure, and since the decane skeleton is connected with a benzoyl substituent, $m / z 121$ fragments were generated here. Combining the fragments $m / z 503[\mathrm{M}+\mathrm{Na}]^{+}$appearing in positive ion mode, the literature suggests that compound 26 is peoniflorin.

Compounds 43 and 44 showed the same molecular weight at $584 \mathrm{Da}$ and almost consistent tandem MS behavior with paeonolide. The fragment ions in the mass spectrum were $m / z 629,553$, and 431. The fragment ion $\mathrm{m} / z 629$ was the molecular ion peak $(\mathrm{m} / z 583$ $\left.[\mathrm{M}-\mathrm{H}]^{-}\right)$plus one molecule of formic acid $(46 \mathrm{u})$, and $\mathrm{m} / \mathrm{z} 553$ was the molecule. The ion peak (m/z $\left.583[\mathrm{M}-\mathrm{H}]^{-}\right)$loses the formation of a molecule of formaldehyde $(30 \mathrm{u})$ on the 6-carbon residue of the glucosyl group, and the fragment ion $\mathrm{m} / \mathrm{z} 553$ further loses a molecule of benzoic acid (122 u), resulting in $\mathrm{m} / z 431$ fragment ions. Positive ion mode yielded $\mathrm{m} / \mathrm{z} 585[\mathrm{M}+\mathrm{H}]^{+}$and $m / z 607[\mathrm{M}+$ $\mathrm{Na}]^{+}$, which identified compound 44 as benzoyl glycoside according to the literature.

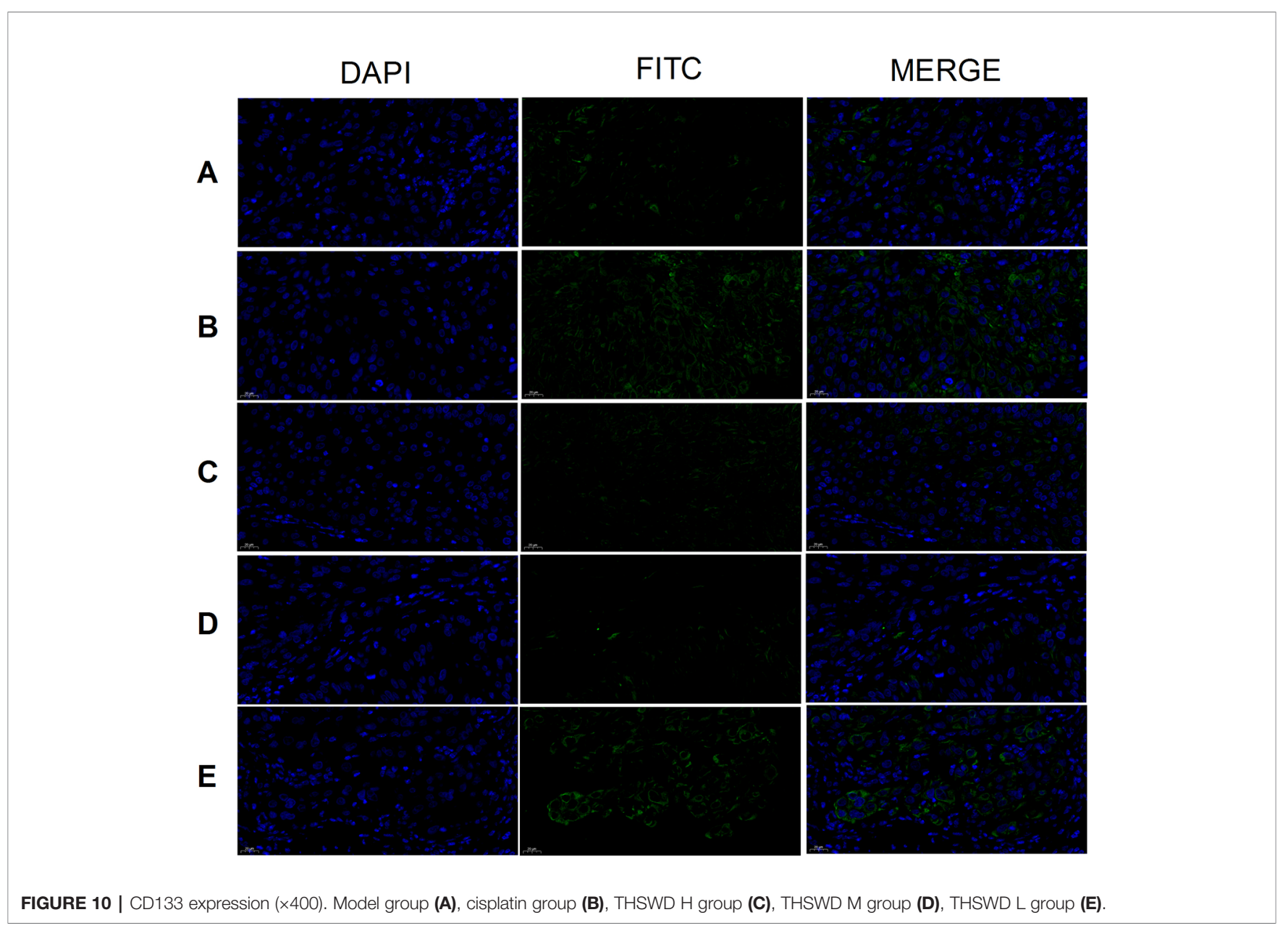




\section{Aromatic Cyanogenic Glycosides}

Most of the common cyanogenic glycosides have the same skeleton structure. According to the substituents on the cyanohydrin derivatives, they can be divided into two categories: aliphatic and aromatic. A given species of plant generally contains only one or two cyanogenic glycosides, such as Rosaceae. Compounds 14 and 15 were all derived from dry mature seeds of the Rosaceae plant Prunus persica (L.) Batsch or Prunus davidiana (Carr.) Franch.

\section{Others}

THSWD also contains human essential amino acids, vitamins, and other ingredients.

\section{H\&E}

$\mathrm{H} \& \mathrm{E}$ stained samples for each group are shown in Figure 7. Tumor cells in the model group were arranged closely, with less necrosis and a large nuclear-to-plasma ratio. The cell morphology was abnormal, and there was more vascular filling. Compared with the model group, tumor cells in the THSWD groups and cisplatin group showed large areas of necrosis with obvious cell debris. The necrotic cells were loosely arranged with large gaps. The results indicate that THSWD exerts a destructive effect on mouse breast cancer cells. This is the first evidence that THSWD has a significant therapeutic effect on breast cancer tumor cells.

\section{IL-4, IL-10, IL-13, and TGF- $\beta 1$ Expression}

To explore the mechanism of THSWD on breast cancer, levels of IL-6, IL- 8 , and IL- $1 \beta$ were measured by ELISA (Table 3 ). IL-5 was significantly increased in the model group. After treatment with THSWD or cisplatin, IL-5 was significantly reduced. The results indicate that THSWD may regulate immune function in breast cancer mice.

\section{CD44, CD117, and CD133 Expression}

Tumor cell CD44 expression was highest in the model group and lowest in the cisplatin group (Figure 8). There was no obvious difference between the three THSWD dose groups. The results showed that cisplatin and THSWD significantly inhibited CD44 expression in tumor cells of breast cancer mice.

CD117 expression was highest in the tumor cells of the model group and lowest in the cisplatin and THSWD middle-dose groups (Figure 9). The results showed that cisplatin and THSWD significantly inhibited CD117 expression in tumor cells of breast cancer mice.

The expression of CD133 was highest in tumor cells of the model group and lowest in the cisplatin group (Figure 10). Results were similar for the THSWD high-, middle-, and lowdose groups. Both cisplatin and THSWD significantly inhibited CD133 expression in tumor cells of breast cancer mice.

\section{REFERENCES}

Chen, Q., and Luo, B. (2018). Study on the naming characteristics of typhoid prescription in Qianjin Fang. J. Shandong Univ. Tradit. Chin. Med. 42 (1), 66-69.

\section{CONCLUSION}

In this study, UPLC-Q-TOF-MS was used for the full chemical characterization of THSWD in both positive and negative ion modes. A total of 87 compounds were identified in 32 minutes, including aromatic acids, flavones, polysaccharides, volatile oils, monoterpene glycosides, aromatic cyanogenic glycosides, and others. This method and the chemical material findings may provide useful information for quality control when producing THSWD and related individual herbs, despite the ingredient complexity and specificity for TCM. The results can be used to develop a quality standard of the TCM compound THSWD and provide a theoretical basis for further study of THSWD metabolism in vivo to clarify the material basis and mechanism of its clinical application.

\section{DATA AVAILABILITY STATEMENT}

The raw data supporting the conclusions of this article will be made available by the authors, without undue reservation, to any qualified researcher.

\section{ETHICS STATEMENT}

All experiments were subject to approval by the Committee on the Ethics of Animal Experiments of Anhui University of Chinese Medicine.

\section{AUTHOR CONTRIBUTIONS}

$\mathrm{XD}$ is responsible for designing the experiment private. LP is responsible for article writing. $\mathrm{QB}$ is responsible for the experimental operation. DP is responsible for data analysis.

\section{FUNDING}

This research was supported by the National Natural Science Fund Regional Innovation and Development Joint Fund Project (No. U19A2009), Anhui University Collaborative Innovation Project (GXXT-2019-043), the Anhui Provincial College Natural Science Research Key Project (No. KJ2019A0466), Excellent and Top Talents Program in Colleges and Universities (No. gxyq2019034), Anhui Provincial Key Laboratory of Traditional Chinese Medicine Compounds (2019AKLCMF03), Open Project of National Key Clinical Specialty (Traditional Chinese Medicine Surgery) (2019zdzk04) and the Natural Science Research Project of Colleges and Universities in Anhui Province (2019fyyb038).

Cheng, L., Wan, H., Fang, Y., Wang, H., Yang, J., and Wan, H. (2018). Mechanism of treating ischemic stroke with Yangyin Yiqi Huoxue therapy. Chin. J. Emerg. Med. 27 (1), 94-96.

Ding, Y., and Zhang, Q. (2010). Progress in clinical and experimental research of Taohong Siwu Decoction. Jiangsu J. Tradit. Chin. Med. 42 (1), 77-79. 
Feng, Y., Chen, Y., and Xin, H. (2017). Analysis of flavonoids in rosae laevigatae fructus by UPLC-Q-TOF-MS. Chin. J. Exp. Formulaics. 23 (12), 71-76.

Gumustas, M., Uslu, B., Ozkan, S. A., and Aboul-Enien, H. Y. (2014). Validated stability-indicating HPLC and UPLC assay methods for the determination of entacapone in pharmaceutical dosage forms. Chromatographia 77, 1721-1726. doi: 10.1007/s10337-014-2758-x

Han, J., Ye, M., Guo, H., Yang, M., Wang, B. R., and Guo, D. A. (2007). Analysis of multiple constituents in a Chinese herbal preparation Shuang-Huang-Lian oral liquid by HPLC-DAD-ESI-MSn. J. Pharm. Biomed. Anal. 44, 430-438. doi: 10.1016/j.jpba.2007.02.023

Hedaya, M. A., Thomas, V., Abdel-Hamid, M. E., Kehinde, E. O., and Phillips, O. A. (2017). A validated UPLC-MS/MS method for the analysis of linezolid and a novel oxazolidinone derivative (PH027) in plasma and its application to tissue distribution study in rabbits. J. Chromatogr. B. Analyt. Technol. Biomed. Life Sci. 1040, 89-96. doi: 10.1016/j.jchromb.2016.11.034

Huang, W. H., and Song, C. Q. (2001). Chemical and pharmacological advances of the study on Angelica sinensis. China J. Chin. Mater. Med. 26, 147-151.

Jiang, H., Rong, R., and Lu, Q. (2011). Liquid chromatography - mass spectrometry identification of Rhubarb chemical composition. Lishizhen Med. Mater. Med. Res. 22 (7), 1705.

Kim, J., Fann, D. Y., Seet, R. C., Jo, D. G., Mattson, M. P., and Arumugam, T. V. (2016). Phytochemicals in ischemic stroke. Neuromol. Med. 18 (3), 283-305.

Li, J., Lu, C., Jiang, M., Niu, X., Guo, H., Li, L., et al. (2012). Traditional Chinese medicine-based network pharmacology could lead to new multicompound drug discovery. Evid. Based Complement. Alternat. Med. 2012, 149762. doi: $10.1155 / 2012 / 149762$

Li, P. L., Liu, M. H., Hu, J. H., and Su, W. W. (2014). Systematic chemical profiling of Citrus grandis 'Tomentosa' by ultra-fast liquid chromatography/diode-array detector/quadrupole time-of-flight tandem mass spectrometry. J. Pharm. Biomed. Anal. 90, 167-179. doi: 10.1016/j.jpba.2013.11.030

Lin, L. Z., He, X. G., Lian, L. Z., King, W., and Elliott, J. (1998). Liquid chromatographic electrospray mass spectrometric study of the phthalides of Angelica sinensis and chemical changes of Z-ligustilide. J. Chromatogr A. 810, 71-79. doi: 10.1016/S0021-9673(98)00201-5

Liu, L., Duan, J. A., Su, S. L., Liu, P., Tang, Y. P., and Qian, D. W. (2015). Research progress of Taowu Siwu Decoction for Siwu Decoction for gynecological blood stasis syndrome dysmenorrhea. Zhongguo Zhong Yao Za Zhi. 40 (5), 814-821.

Liu, M. J., Wang, Y., Li, L., Li, X., Dai, Y., Zhang, C., et al. (2017). UPLC-Q-TOFMS/MS rapid identification analysis of chemical composition of Sanhuang Tablet. China J. Chin. Mater. Medica. 42 (9), 1685-1692. doi: 10.19540/ j.cnki.cjcmm.20170223.010

Lu, X. H., Zhang, J. J., Liang, H., and Zhao, Y. Y. (2004). Chemical constituents of Angelica sinensis. J. Chin. Pharm. Sci. 13, 1-3. doi: 10.1097/TP.0b013e3181821c25

Ma, Y. L., Li, Q. M., Van den Heuvel, H., and Claeys, M. (1997). Characterization of flavone and flavonolaglycones by collision-induced dissociation tandem mass spectrometry. Rapid Commun. Mass Spectrom. 11 (12), 1357-1364. doi: 10.1002/(SICI)1097-0231(199708)11:12<1357::AID-RCM983>3.0.CO;2-9

Ning, Y., Zeng, S., Peng, G., and Wang, D. (2012). Content determination of hydroxysafflor yellow A in Tibetan Medicine Shisanwei Honghua Pills by LCMS/MS. Chin. Pharm. 23 (27), 2538-2541.

Sun, H., Zhu, C., Zhang, H., Wang, Y., Luo, G., Hu, P., et al. (2009). Rhubarb and its processed products of liquid chromatography and mass analysis and material basis comparison. Chin. Patent Med. 31 (3), 421.

Wang, Y., and Han, Y. (2011). Study on Fingerprint of ligusticun chuanxiong Hort by HPLC. Chin. Pharm. Aff. 25 (10), 1017-1021.

Weiwei, H. (2017). Thoroughly and comprehensively study and understand the "Chinese Medicine Law" to support the development of traditional chinese medicine in accordance with the law. J. Nanjing Univ. Chin. Med. (Soc. Sci. Edition). 18 (1), 6-13.

Wu, J., Fang, X., Yuan, Y., Dong, Y., Liang, Y., Xie, Q., et al. (2017). UPLC/Q-TOFMS profiling of phenolics from Canarium pimela leaves and its vasorelaxant and antioxidant activities. Rev. Bras. Farmacogn. 27 (6), 716-723. doi: 10.1016/ j.bjp.2017.10.005

Yang, L., Xie, X., Wan, L., Wang, S. B., and Zhan, K.. (2007). Chemical constituents from ligusticun chuanxiong Hort. Lishizhen Med. Mater. Med. Res. 18 (7), 1576-1577.

Yu, L., Chen, Y., and Xue, M. (2007). The pathogenesis of ischemic stroke is discussed. Mod. J. Integ. Tradit. Chin. West. Med. 16 (11), 1478-1479.

Zhang, J., Chen, X., and Sun, Y. (2014). Simultaneous determination of hydroxysafflor yellow A, ferulic acid and ammonium glycyrrhizinate in Xinshenghua Granules by RP-HPLC. Chin. Patent Med. 36 (2), 310-313.

Zhang, X., Liang, C., Li, C., Bu, M., Bu, L., Xiao, Y., et al. (2018). Simultaneous qualitative and quantitative study of main compounds in Commelina communis Linn by UHPLC-Q-TOF-MS-MS and HPLC-ESI-MS-MS. $J$. Chromatogr. Sci. 56 (7), 582-594. doi: 10.1093/chromsci/bmy030

Zhang, X. (2010). Discussion on the factors affecting the quality of chinese medicinal materials. Chin. Mod. Doctor. 48 (16), 54-55.

Zhen, H. (2013). Connotation and extension of Chinese medicine culture. J. Tradit. Chin. Med. 54 (3), 192-194.

Conflict of Interest: The authors declare that the research was conducted in the absence of any commercial or financial relationships that could be construed as a potential conflict of interest.

Copyright $\odot 2020$ Duan, Pan, Bao and Peng. This is an open-access article distributed under the terms of the Creative Commons Attribution License (CC BY). The use, distribution or reproduction in other forums is permitted, provided the original author (s) and the copyright owner(s) are credited and that the original publication in this journal is cited, in accordance with accepted academic practice. No use, distribution or reproduction is permitted which does not comply with these terms. 